\title{
ON THE UNIFORM CONVERGENCE OF THE DEVELOPMENTS IN BESSEL FUNCTIONS*
}

BY

\section{CHARLES N. MOORE}

In many problems of mathematical physics we are led to the question of whether or not it is possible to represent an arbitrary function by a series of the form

$$
A_{1}^{\prime} J_{v}\left(\lambda_{1} x\right)+A_{2}^{\prime} J_{v}\left(\lambda_{2} x\right)+\cdots,
$$

where the $A^{\prime \prime}$ s are constants, $J_{\nu}(x)$ represents a Bessel function of order $\nu$, and the $\lambda$ 's are the roots of the equation

$$
l \lambda J_{\nu}^{\prime}(\lambda)+h J_{\nu}(\lambda)=0,
$$

in which $h$ and $l$ are any constants not both zero. In most cases we find that we have a solution of our problem if the $A$ 's can be so determined that:

(a) The series (1) converges uniformly to $f(x)$ throughout any closed interval lying in the interval $0 \leqq x \leqq 1$ and not including a point of discontinuity of $f(x)$, or, in some instances, the origin or the point $x=1$, and

(b) The sum of the first $n$ terms of the series remains finite for all values of $n$ and all values of $x$ lying in the interval $0 \leqq x \leqq 1$.

The ordinary formal method of determining the coefficients of an expansion of an arbitrary function in terms of normal functions, gives us the following value for $A_{n}^{\prime}$

$$
A_{n}^{\prime}=\frac{\int_{0}^{1} x f(x) J_{\nu}\left(\lambda_{n} x\right) d x}{\int_{0}^{1} x\left[J_{\nu}\left(\lambda_{n} x\right)\right]^{2} d x},
$$

where $\lambda_{n}$ is the $n$th positive root of equation (2). This determination of the $A^{\prime \prime} \mathrm{s}$ is found on investigation to give the desired expansion of $f(x)$ except in the case

$$
l \nu+h=0,
$$

when an extra term is required in order that the series may have the proper value.

\footnotetext{
* Presented to the Society, October 30, 1909 and February 25, 1911.
} 
That the case in which (4) is satisfied is really not an exceptional case at all, but only seems so on account of the notation employed, has been pointed out in a previous paper by the writer.* Moreover, as was also brought out in the previous paper, the appearance of an exceptional case can be avoided by introducing the notation

$$
F_{\nu}(\lambda, x) \equiv\left(\begin{array}{l}
x \\
\overline{2}
\end{array}\right)^{\nu} \sum_{i=0}^{\infty} \frac{(-1)^{i}(\lambda x)^{2 i}}{2^{2 i} i ! \Gamma(\nu+i+1)} \equiv \frac{1}{\lambda^{\nu}} J_{\nu}(\lambda x),
$$

and considering the series

$$
\sum_{n=1}^{\infty} F_{\nu}\left(\lambda_{n}, x\right) \frac{\int_{0}^{1} x f(x) F_{\nu}\left(\lambda_{n}, x\right) d x}{\int_{0}^{1} x\left[F_{\nu}\left(\lambda_{n}, x\right)\right]^{2} d x}=\sum_{n=1}^{\infty} A_{n} F_{\nu}\left(\lambda_{n}, x\right),
$$

where the $\lambda$ 's are the successive root's, positive or zero, of the equation

$$
\left[l \frac{\partial}{\partial x} F_{\nu}(\lambda, x)+h F_{\nu}(\lambda, x)\right]_{x=1}=0, \dagger
$$

arranged in increasing order of magnitude. This series is identical with the series (1) where the $\lambda$ 's are the successive positive roots of (2), except in the case where (4) is satisfied, and in that case only differs from it by having an extra term at the beginning. This extra term is finite and continuous, and hence in all questions involving merely convergence or the remaining finite of the sum of $n$ terms of the series, we may deal with series (1), and it is only necessary to consider series (6) when we come to the question of the value of the development.

It has been shown by several writers $\ddagger$ that the series $(6)$ will, under very broad restrictions upon $f(x)$, converge uniformly to $f(x)$ throughout any closed interval, lying in the interval $0<x<1$ and not including a point of discontinuity of $f(x)$. The question of whether or not, under certain restrictions upon $f(x)$, the series (6) will converge to $f(0)$ at the origin, $\S$ and will converge uniformly to $f(x)$ in an interval including the origin and in an inter-

\footnotetext{
* Cf. these Transactions, vol. 10 (1909), p. 420. That the exceptional case was such only on account of the notation employed, and that it would cease to be exceptional if we adopted the proper notation, was pointed out to me by Professor BôchKR, to whose valuable suggestions I. owe many ideas that have been of great use in this as well as in the preceding investigation.

†The positive roots of this equation are obviously identical with the positive roots of equation (2).

†Cf. Drni, Scrie di Fourier (Pisa, 1880), pp. 246-269; Knrser, Mathematisohe Annalen, vol. 63 (1906-07), p. 505; Hobson, Proceedings of the London Mathematical Society, ser. 2 , vol. 7 (1909), p. 359.

$\S$ It is only in the case $v=0$ that this question is anything but a trivial one, since for $v>0$ every term of the series vanishes when $x=0$ and the value of the series is obviously zero for this value of $x$.
} 
val including the point $x=1$ has not been settled in the papers referred to; neither has it been shown that, under suitable restrictions upon $f(x)$, the sum of $n$ terms of the series will remain finite for all values of $n$ and all values of $x$ in the interval $0 \leqq x \leqq 1$. Hilbert has shown* for the particular case where $\nu=0$ and the $l$ of equation (7) is zero that if $f(x)$ vanishes for $x=1$ and satisfies the rather stringent conditions of being continuous, together with its first and second derivatives, throughout the interval $0 \leqq x \leqq 1$, the series (6) will converge uniformly to $f(x)$ throughout the interval $0 \leqq x \leqq 1$.

For most of the applications to mathematical physics it is essential to know something about the behavior of the series (6) in the neighborhood of the origin, of the point $x=1$, and of points of discontinuity of $f(x)$, when such exist, in order to be sure that we really have a solution of the physical problem we are discussing. In the majority of these cases, conditions like HilberT's are too binding, and it is necessary to remove the restriction that the function should have no discontinuity; it is also necessary, even in the particular case that Hilbert treats, to determine the behavior of the series in the neighborhood of the point $x=1$ when $f(1) \neq 0$.

The object of the present paper is to show that in case $f(x)$ is made up of a number of pieces in each of which $f(x)$ and its derivative $f^{\prime}(x)$ are continuous, while $f^{\prime \prime}(x)$ exists, and is finite and integrable, the series (6) will possess the desired properties. These conditions on $f(x)$ are usually met with in the applications; in a later paper the writer proposes to discuss the uniform summability of the series in the neighborhood of $x=0$ and $x=1$, thus showing the existence of a solution of the physical problem under extremely broad conditions. $f$

LEMмA 1. If in the interval $0 \leqq x \leqq 1$, the function $f(x)$ is continuous, save at a finite number of points at which it has a finite jump, if in each interval of continuity it has a first derivative that is continuous, save at a finite number of points at which it has a finite jump, and if finally in each interval of continuity of the first derivative the function has a second derivative that is finite and integrable, the coefficient $A_{n}^{\prime}$ of the general term of the series (1) may be voritten in the form

$$
\begin{aligned}
& \sum_{n=1}^{m=1} \dot{M}_{m} \frac{\cos \left[(n \pi+q) c_{m}-\frac{2 \nu+3}{4} \pi\right]}{\sqrt{n}} \\
&+M f(1) \frac{\cos \left[(n \pi+q)-\frac{2 \nu+3}{4} \pi\right]}{\sqrt{n}}+\frac{\nu K f(0)}{\lambda_{n}}+r_{n},
\end{aligned}
$$

* Göttinger Nachrichten (1904), Grundzïge einer allgemeinen Theorie der linearen Integralgleichungen (Zweite Mitteilung), p. 229. Previously KNESER had discussed a special aase slightly different from the one treated by HiLBert, using similar restrictions upon $f(x)$. (Cf. Archiv der Mathematik und Physik, 3rd ser., vol. 7 (1904), pp. 123-133.)

$\dagger$ Ct. the introduction to the paper in these Transactions referred to above.

Trans. Am. Math. Soc. 13 
where the $M$ 's and $K$ are constants, the c's are the points of discontinuity of $f(x)$, and $r_{n}$ is the general term of an absolutely convergent series.

We have *

$$
\int_{0}^{01} x\left[J_{\nu}\left(\lambda_{n} x\right)\right]^{2} d x=\frac{A^{2}}{2 \lambda_{n}}+\frac{\psi_{1}\left(\lambda_{n}\right)}{\lambda_{n}^{2}},
$$

where $A$ is a constant, and $\psi_{1}\left(\lambda_{n}\right)$ is used to represent any function of $\lambda_{n}$ that remains finite when $\lambda_{n}$ increases indefinitely. Hence it follows from (3) that if we subtract from $A_{n}^{\prime}$ the term

$$
\frac{2 \lambda_{n}}{A^{2}} \int_{0}^{1} x f(x) J_{\nu}\left(\lambda_{n} x\right) d x
$$

we shall have left the term

$$
\frac{\frac{-2 \psi_{1}\left(\lambda_{n}\right)}{A^{2} \lambda_{n}} \int_{0}^{1} x f(x) J_{v}\left(\lambda_{n} x\right) d x}{\frac{A^{2}}{2 \lambda_{n}}+\frac{\psi_{1}\left(\lambda_{n}\right)}{\lambda_{n}^{2}}} .
$$

We will show first that (11) is the general term of an absolutely convergent series. Then, that by subtracting from (10) quantities that are the general terms of absolutely convergent series, we can reduce it to the sum of the first three terms in (8). Our lemma will thus be established.

Let us use $\phi_{m}(x)$ to represent a function that is continuous in the interval $0 \leqq x \leqq c_{m+1}$, that has in this interval a first derivative that is continuous, save at a finite number of points at which it has a finite jump, and a second derivative that is finite and integrable, and furthermore is such that

$$
\phi_{m}(x)=f(x) \quad\left(c_{m} \leqq x \leqq c_{m+1} ; m=0,1,2, \cdots, k\right),
$$

where for the sake of uniformity we have set

Then we may write

$$
c_{0}=0, \quad c_{k+1}=1 .
$$

$$
\begin{aligned}
\int_{0}^{1} x f(x) J_{\nu}\left(\lambda_{n} x\right) d x=\sum_{m=0}^{m=k} \int_{0}^{c_{m+1}} x \phi_{m}(x) & J_{\nu}\left(\lambda_{n} x\right) d x \\
& -\sum_{m=1}^{m=k} \int_{0}^{c_{m}} x \phi_{m}(x) J_{\nu}\left(\lambda_{n} x\right) d x .
\end{aligned}
$$

But if $c$ is a constant lying in the interval $0<x<1$, and $\phi(x)$ is a function that is continuous in the interval $0 \leqq x \leqq c$ and has a first derivative that is continuous in this interval save at a finite number of points, we have, $\dagger$ if we set $\lambda_{n} x=y$ and integrate by parts,

* Ct. equation (46) o: the writer's article in these Transactions, just referred to.

†Cf. ByerLy, An Elementary Treatise on Fourier's Series, etc., p. 223, formula (4). 


$$
\begin{aligned}
& \int_{0}^{c} x \phi(x) J_{\nu}\left(\lambda_{n} x\right) d x=\frac{1}{\lambda_{n}^{2}} \int_{0}^{c \lambda_{n}} y^{v+1} J_{v}(y) \frac{1}{y^{\nu}} \phi\left(\frac{y}{\lambda_{n}}\right) d y \\
&=\frac{1}{\lambda_{n}^{2}}\left[y^{v+1} J_{v+1}(y) \frac{1}{y^{\nu}} \phi\left(\frac{y}{\lambda_{n}}\right)\right]_{0}^{c \lambda_{n}} \\
&-\frac{1}{\lambda_{n}^{2}} \int_{0}^{c \lambda_{n}} y^{v+1} J_{v+1}(y)\left\{-\frac{\nu}{y^{v+1}} \phi\left(\frac{y}{\lambda_{n}}\right)+\frac{1}{\lambda_{n} y^{\nu}} \phi^{\prime}\left(\frac{y}{\lambda_{n}}\right)\right\} d y \\
&= \frac{1}{\lambda_{n}} c J_{v+1}\left(c \lambda_{n}\right) \phi(c)+\frac{\nu}{\lambda_{n}^{2}} \int_{0}^{c \lambda_{n}} J_{v+1}(y) \phi\left(\frac{y}{\lambda_{n}}\right) d y \\
&-\frac{1}{\lambda_{n}^{3}} \int_{0}^{c \lambda_{n}} y J_{v+1}(y) \phi^{\prime}\left(\frac{y}{\lambda_{n}}\right) d y \\
&= \frac{c \phi(c) J_{v+1}\left(c \lambda_{n}\right)}{\lambda_{n}}+\frac{\nu}{\lambda_{n}} \int_{0}^{c} J_{v+1}\left(\lambda_{n} x\right) \phi(x) d x \\
&-\frac{1}{\lambda_{n}} \int_{0}^{c} x J_{v+1}\left(\lambda_{n} x\right) \phi^{\prime}(x) d x .
\end{aligned}
$$

Moreover, we have from the asymptotic expansion * of $J_{v+1}(x)$

$$
J_{v+1}\left(\lambda_{n} x\right)=\frac{\psi\left(\lambda_{n} x\right)}{\lambda_{n} x^{l}},
$$

where ve use $\psi(x)$ to represent any function of $x$ that is continuous for all values of $x>0$, remains finite for all such values of $x$, and approaches $a$ finite limit when $x$ approaches zero.

From (14) and (15) we have

$$
\left|\int_{0}^{c} x \phi(x) J_{v}\left(\lambda_{n} x\right) d x\right|<\frac{K_{1}}{\lambda_{n}}
$$

where $K_{1}$ is a positive constant. Combining (16) and (13), we obtain

$$
\left|\int_{0}^{1} x f(x) J_{\nu}\left(\lambda_{n} x\right) d x\right|<\frac{K_{2}}{\lambda_{n}^{l}},
$$

where $K_{2}$ is a positive constant. But for values of $\boldsymbol{n}$ so great that

$$
\left|\frac{\Psi_{1}\left(\lambda_{n}\right)}{\lambda_{n}^{2}}\right|<\frac{A^{2}}{4 \lambda_{n}},
$$

the quantity (11) is less in absolute value than

$$
\frac{8\left|\psi_{1}\left(\lambda_{n}\right)\right|}{A^{4}} \cdot\left|\int_{0}^{1} x f(x) J_{v}\left(\lambda_{n} x\right) d x\right|,
$$

* Cf. Lipschitz, Crelle's Journal, vol. 56 (1859), pp. 193-196. 
and hence, in view of (17), is less in absolute value than $K_{3} / \lambda_{n}^{\frac{3}{3}}$, where $K_{3}$ is a positive constant. Consequently, since a positive constant $C$ exists such that*

$$
\lambda_{n}>C n,
$$

it follows that (11) is the general term of an absolutely convergent series.

It remains to consider the term (10) which was subtracted from $A_{n}^{\prime}$ to produce (11). We must first, however, discuss the last two terms on the right hand side of equation (14).

The asymptotic expansion of $J_{\nu+1}(x)$ gives

$$
J_{\nu+1}\left(\lambda_{n} x\right)=\sqrt{\frac{2}{\pi \lambda_{n} x}} \cos \left(\lambda_{n} x-\frac{2 \nu+3}{4} \pi\right)+\frac{\psi\left(\lambda_{n} x\right)}{\lambda_{n}^{3} x^{3}}
$$

Substituting this value in the integral of the third term on the right hand side of (14), we obtain

$$
\begin{array}{r}
\int_{0}^{c} x \phi^{\prime}(x) J_{v+1}\left(\lambda_{n} x\right) d x=\frac{A}{\sqrt{\lambda_{n}}} \int_{0}^{c} \sqrt{x} \phi^{\prime}(x) \\
\end{array}
$$

where for simplicity we have used $A$ and $\alpha$ in place of $\sqrt{2 / \pi}$ and $(2 \nu+3) \pi / 4$.

If $\phi(x)$ is a function whose first derivative is continuous in the interval $0 \leqq x \leqq c$, except at a finite number of points at which it has a finite jump, and whose second derivative is finite and integrable there, we have from an integration by parts

$$
\begin{aligned}
\int_{0}^{c} \sqrt{x} \phi^{\prime}(x) \cos \left(\lambda_{n} x-\alpha\right) & d x=\frac{1}{\lambda_{n}} \sum_{i=1}^{i=r+1}\left[\sqrt{k_{i}} \phi^{\prime}\left(k_{i}-0\right) \sin \left(\lambda_{n} k_{i}-\alpha\right)\right. \\
& \left.-\sqrt{k_{i-1}} \phi^{\prime}\left(k_{i-1}+0\right) \sin \left(\lambda_{n} k_{i-1}-\alpha\right)\right] \\
& -\frac{1}{\lambda_{n}} \int_{0}^{c}\left[\sqrt{x} \phi^{\prime \prime}(x)+\frac{\phi^{\prime}(x)}{2 \sqrt{2}}\right] \sin \left(\lambda_{n} x-\alpha\right) d x,
\end{aligned}
$$

where $k_{1}, k_{2}, \cdots, k_{r}$ are the points of discontinuity of $\phi^{\prime}(x)$, and for the sake of uniformity we have set

$$
k_{0}=0, \quad k_{r+1}=c .
$$

Combining (20) and (21), we have

If we set

$$
\int_{0}^{c} x \phi^{\prime}(x) J_{\nu+1}\left(\lambda_{n} x\right) d x=\frac{\psi_{1}\left(\lambda_{n}\right)}{\lambda_{n}^{3}} .
$$

$$
F(x)=\frac{\phi(x)-\phi(0)}{x}
$$

${ }^{*}$ Cf. equation (23) of the writer's article in these Transactions, referred to above. 
we obtain for the integral in the second term on the right hand side of (14)

$$
\int_{0}^{c} J_{\nu+1}\left(\lambda_{n} x\right) \phi(x) d x=\phi(0) \int_{0}^{c} J_{\nu+1}\left(\lambda_{n} x\right) d x+\int_{0}^{c} x F(x) J_{\nu+1}\left(\lambda_{n} x\right) d x
$$

If we substitute in the second term on the right hand side of (24) the value of $J_{v+1}\left(\lambda_{n} x\right)$ given by $(19)$, we get

$$
\begin{aligned}
\int_{0}^{c} x F(x) J_{\nu+1}\left(\lambda_{n} x\right) d x=\frac{A}{\sqrt{\lambda_{n}}} \int_{0}^{c} \sqrt{x} \cos \left(\lambda_{n} x-\alpha\right) F(x) d x \\
+\frac{1}{\lambda_{n}^{\frac{3}{2}}} \int_{0}^{c} \frac{\psi\left(\lambda_{n} x\right)}{\sqrt{x}} F(x) d x,
\end{aligned}
$$

where again, for the sake of simplicity, we have introduced $A$ and $\alpha$ in place of $\sqrt{2 / \pi}$ and $(2 \nu+3) \pi / 4$. By means of an integration by parts, the integral in the first term on the right hand side of (25) reduces to the form

$$
\frac{\sin \left(\lambda_{n} c-\alpha\right)}{\lambda_{n}} \sqrt{c} F(c)-\frac{1}{\lambda_{n}} \int_{0}^{c}\left[\sqrt{x} F^{\prime}(x)+\frac{F(x)}{2 \sqrt{x}}\right] \sin \left(\lambda_{n} x-\alpha\right) d x
$$

Substituting this expression in (25), we obtain for the second term on the right band side of (24)

$$
\int_{0}^{c} x F(x) J_{v+1}\left(\lambda_{n} x\right) d x=\frac{\psi_{1}\left(\lambda_{n}\right)}{\lambda_{n}^{l}}
$$

If we set $\lambda_{n} x=y$, we get for the integral in the first term on the right hand side of (24)

$$
\int_{0}^{c} J_{v+1}\left(\lambda_{n} x\right) d x=\frac{1}{\lambda_{n}} \int_{0}^{c \lambda_{n}} J_{v+1}(y) d y
$$

where the integral on the right hand side may be written

$$
\int_{0}^{\infty} J_{\nu+1}(y) d y-\int_{c \lambda_{n}}^{\infty} J_{\nu+1}(y) d y,
$$

since these integrals converge.

Making use of (19), we obtain for the second term in (29)

$$
\int_{c \lambda_{n}}^{\infty} J_{\nu+1}(y) d y=A \int_{c \lambda_{n}}^{\infty} \frac{\cos (y-\alpha)}{\sqrt{y}} d y+\int_{c \lambda_{n}}^{\infty} \frac{\Psi(y)}{y^{2}} d y
$$

where this reduction is legitimate since the two integrals on the right hand side of $(30)$ both converge. For the second of these integrals we have

$$
\left|\int_{c \lambda_{n}}^{\infty} \psi(y) d y\right|<N \int_{c \lambda_{n}}^{\infty} \frac{d y}{y^{\frac{3}{2}}} d y=\frac{N}{c^{\frac{3}{2}} \lambda_{n}^{\frac{1}{2}}},
$$

where $N$ is a positive constant. For the first integral on the right hand side of (30) we have from an integration by parts 


$$
\int_{c \lambda_{n}}^{\infty} \frac{\cos (y-\alpha)}{\sqrt{y}} d y=\left[\frac{\sin (y-\alpha)}{\sqrt{y}}\right]_{c \lambda_{n}}^{\infty}+\frac{1}{2} \int_{c \lambda_{n}}^{\infty} \frac{\sin (y-\alpha)}{y^{2}} d y=\frac{\psi_{1}\left(\lambda_{n}\right)}{\lambda_{n}}
$$

Combining (31) and (32) with (30), we obtain

$$
\int_{c \lambda_{n}}^{\infty} J_{\nu+1}(y) d y=\frac{\psi_{1}\left(\lambda_{n}\right)}{\lambda_{n}^{\frac{1}{2}}}
$$

Substituting (33) in (29), and remembering that (29) is only another way of writing the integral on the right hand side of (28), we get

$$
\int_{0}^{c} J_{v+1}\left(\lambda_{n} x\right) d x=\frac{K^{\prime}}{\lambda_{n}}+\frac{\psi_{1}\left(\lambda_{n}\right)}{\lambda_{n}^{\prime}},
$$

where for the sake of simplicity we have used $K^{\prime}$ in place of the first term in (29).

Combining (24), (27), and (34), we obtain

$$
\int_{0}^{c} J_{v+1}\left(\lambda_{n} x\right) \dot{\phi}(x) d x=\frac{K^{\prime} \phi(0)}{\lambda_{n}}+\frac{\psi_{1}\left(\lambda_{n}\right)}{\lambda_{n}^{i}}
$$

From (14), (22), and (35), we get

$$
\int_{0}^{c} x \phi(x) J_{v}\left(\lambda_{n} x\right) d x=\frac{c \phi(c) J_{\nu+1}\left(c \lambda_{n}\right)}{\lambda_{n}}+\frac{\nu K^{\prime} \phi(0)}{\lambda_{n}^{2}}+\frac{\psi_{1}\left(\lambda_{n}\right)}{\lambda_{n}^{!}}
$$

Finally from (13) and (36), we obtain

$$
\begin{aligned}
\int_{0}^{1} x f(x) J_{\nu}\left(\lambda_{n} x\right) d x & =\sum_{m=0}^{m=k} \frac{c_{m+1} \phi_{m}\left(c_{m+1}\right) J_{\nu+1}\left(c_{m+1} \lambda_{n}\right)}{\lambda_{n}} \\
& -\sum_{m=1}^{m=k} \frac{c_{m} \phi_{m}\left(c_{m}\right) J_{\nu+1}\left(c_{m} \lambda_{n}\right)}{\lambda_{n}}+\frac{\nu K^{\prime} f(0)}{\lambda_{n}^{2}}+\frac{\psi_{1}\left(\lambda_{n}\right)}{\lambda_{n}} .
\end{aligned}
$$

It follows from (37) that by subtracting from (10) a quantity that is the general term of an absolutely convergent series, we may reduce it to the form

$$
\frac{2}{A^{2}} \sum_{m=0}^{m=k} c_{m+1} \phi_{m}\left(c_{m+1}\right) J_{\nu+1}\left(c_{m+1} \lambda_{n}\right)-\frac{2}{A^{2}} \sum_{m=1}^{m=k} c_{m} \phi_{m}\left(c_{m}\right) J_{\nu+1}\left(c_{m} \lambda_{n}\right)+\frac{\nu K f(0)}{\lambda_{n}}
$$

where we have replaced $2 K^{\prime} / A^{2}$ by $K$. If, now, we can show that by subtracting from (38) a quantity that is the general term of an absolutely convergent series we may reduce it to the sum of the first three terms in (8), our lenma will be proved.

We have [loc. cit., equation (58)]

$$
\text { (39) } \cos \left(\lambda_{n} c-\frac{(2 \nu+3) \pi}{4}\right)=\cos \left[(n \pi+q) c-\frac{(2 \nu+3) \pi}{4}\right]+\frac{\psi_{1}\left(\lambda_{n}\right)}{n} \text {. }
$$


Combining (39) with (19) and (18), we obtain

$$
J_{v+1}\left(\lambda_{n} c\right)=\sqrt{\frac{2}{\pi \lambda_{n} c}} \cos \left[(n \pi+q) c-\frac{(2 \nu+3) \pi}{4}\right]+\frac{\psi_{1}\left(\lambda_{n}\right)}{n^{!}} .
$$

But since [loc. cit. equation (57)]

$$
\lambda_{n}=n \pi+q+\frac{\Psi_{1}\left(\lambda_{n}\right)}{n}
$$

we have by (18)

$$
\frac{1}{\sqrt{\lambda_{n}}}-\frac{1}{\sqrt{n \pi}}=\frac{n \pi-\lambda_{n}}{\sqrt{\lambda_{n} n \pi}\left(\sqrt{\lambda_{n}}+\sqrt{n \pi}\right)}=\frac{\psi_{1}(n)}{n^{\prime}} .
$$

Combining (40) and (42), we obtain

$$
J_{v+1}\left(\lambda_{n} c\right)=\frac{\sqrt{2} \cos \left[(n \pi+q) c-\frac{2 \nu+3}{4} \pi\right]}{\pi \sqrt{n c}}+\frac{\psi_{1}(n)}{n !}
$$

It follows at once from (43) that by subtracting from (38) a quantity that is the general term of an absolutely convergent series, we can reduce it to the sum of the first three terms in (8). Our lemma is therefore established.

Before beginning the proof of the next lemma, we shall state a few formulse connected with Bessel functions, which will be useful in the course of that proof.

We have [loc. cit. equation (58)]

$$
\cos \left(\lambda_{n} x-\alpha\right)=\cos (n \pi x+q x-\alpha)+\frac{\psi\left(x, \lambda_{n}\right)}{n},
$$

where $\lambda_{n}$ is the $n$th positive root of equation (2) and

$$
\left|\Psi\left(x, \lambda_{n}\right)\right|<K_{1} \quad(0 \leqq x \leqq 1 ; n=1,2,3, \cdots),
$$

$K_{1}$ being a positive constant.

From the asymptotic expansion * we have

$$
J_{\nu}\left(\lambda_{n} x\right)=\frac{A}{\sqrt{\lambda_{n} x}} \cos \left(\lambda_{n} x-\frac{(2 \nu+1) \pi}{4}\right)+\frac{\psi\left(\lambda_{n} x\right)}{\left(\lambda_{n} x\right)^{1}},
$$

where

$$
|\psi(x)|<K_{2}, \quad\left|\psi^{\prime}(x)\right|<K_{3}
$$

$K_{2}$ and $K_{3}$ being positive constants.

* Cf. LIPschitz, loo. cit. That the second inequality in (47) holds may be seen by differentiating the expression of the form (47) for $J_{\nu}(x)$ and comparing the result with the value of $J_{v}^{\prime}(x)$ obtained by combining the equation

$$
J_{\nu}^{\prime}(x)=-J_{v+1}(x)+\frac{\nu}{x} J_{v}(x)
$$

with the expression of the form (46) for $J_{\nu}(x)$ and the expression of the form (19) for $J_{\nu+1}(x)$. 
Lемма 2. The quantity

$$
\frac{\cos \left[(n \pi+q) c-\frac{2 \nu+3}{4} \pi\right] J_{\nu}\left(\lambda_{n} x\right)}{\sqrt{n}}
$$

where $c$ is any positive constant $\leqq 1$, is the general term of a series that is uniformly convergent in any interval

$$
0 \leqq x \leqq x_{0}<c .
$$

The quantity (48) may be written in the form

$$
\begin{aligned}
\cos \left(q c-\frac{2 \nu+3}{4} \pi\right) \frac{\cos n \pi c J_{\nu}\left(\lambda_{n} x\right)}{\sqrt{n}} & \\
& -\sin \left(q c-\frac{2 \nu+3}{4} \pi\right) \frac{\sin n \pi c J_{\nu}\left(\lambda_{n} x\right)}{\sqrt{n}},
\end{aligned}
$$

and hence if we can show that each of the quantities

$$
\frac{\cos n \pi c J_{\nu}\left(\lambda_{n} x\right)}{\sqrt{n}}, \quad \frac{\sin n \pi c J_{\nu}\left(\lambda_{n} x\right)}{\sqrt{n}},
$$

is the general term of a series that is uniformly convergent in the interval (49), our lemma will be proved.

Let us consider the series whose general term is the first expression in (51); the proof for the series whose general term is the second expression in (51) is entirely analogous. Since $0<c<2$, the series that we are discussing converges for $x=0$;* it remains to be shown that it converges uniformly in the interval $0<x \leqq x_{0}<c$.

Given $\delta$, positive and arbitrarily small, let us choose $m$ such that $\dagger$

$$
\begin{array}{cr}
\left|\sum_{n=p}^{n=q \sin (n \pi z)} \frac{\delta}{\sqrt{n}}\right|<\eta=\frac{\delta}{1+C_{2}+C_{3}} & \left(\begin{array}{r}
c-x_{0} \leqq z \leqq c+x_{0} \\
q>p \geqq m
\end{array}\right), \\
\sum_{n=p}^{n=q} \frac{1}{n^{\frac{3}{2}}}<\eta & (q>p \geqq m),
\end{array}
$$

where

$$
C_{2}=\frac{A}{\sqrt{k}}\left(K_{1}+4\right), \quad C_{3}=\frac{2^{\frac{3}{2}} N^{\frac{1}{2}}\left(3 C K_{3} k+2 K_{2} N\right)+6 K_{2} C^{\frac{5}{2}}}{3 C^{\frac{6}{2}} k_{2}},
$$

the symbols $A, C, K_{1}, K_{2}, K_{3}$ representing the same quantities as in equations

* For $v>0$ all the terms of the series are zero, and its convergence is obvious; for $v=0$, cf. Schlömich, Algebraische Analysis, 3d ed., 1862, §31, also Bûcher, Annals of Mathematics, ser. 2, vol. 7 (1906), pp. 109-111.

† That the inequality (52) holds for sufficiently large values of $m$ is evident from a reference to page 111 of the article by BôcHER, quoted in the previous footnote. 
(46), (18), (45), and (47); $k$ is the smallest positive root of $J_{\nu}^{\prime}(x)=0$, and $N$ is a positive constant such that *

$$
\lambda_{n}<N n \text {. }
$$

Let 8 be the greatest integer such that $\lambda_{f} x<k$, and $\mu$ and $\nu$ any two integers such that

Three cases must be considered :

$$
m \leqq \mu \leqq \nu
$$

$$
\text { (A) } \mu \leqq s<\nu, \quad(B) \quad \nu \leqq s, \quad(C) \mu>s .
$$

Beginning with $(A)$, we write

$$
\sum_{n=\mu}^{n=\nu} \frac{\cos n \pi c J_{\nu}\left(\lambda_{n} x\right)}{V^{\prime} \bar{n}}=\sum_{n=\mu}^{n=s}+\sum_{n=s+1}^{n=\nu}=R_{1}+R_{2} .
$$

From (52) and a lemma due to ABEL, $\nmid$ we have

For $R_{2}$ we have from $(46) \ddagger$

$$
\left|R_{1}\right|<\eta \quad\left(0<x \leqq x_{0}\right)
$$

$$
R_{2}=A \sum_{i+1}^{\nu} \frac{\cos n \pi c \cos \left(\lambda_{n} x-\alpha\right)}{\sqrt{n \lambda_{n} x}}+\sum_{s+1}^{\nu} \frac{\cos n \pi c \psi\left(\lambda_{n} x\right)}{\sqrt{n \lambda_{n}^{3} x^{3}}}
$$

The general term of the first series on the right hand side of (58) may, in view of (44), be written in the form

$$
\frac{\cos n \pi c \cos (n \pi x+q x-\alpha)}{\sqrt{n \lambda_{n} x}}+\frac{\cos n \pi c \psi\left(x, \lambda_{n}\right)}{n \sqrt{n \lambda_{n} x}} .
$$

From (45), (53), and the fact that 8 was so chosen that $\lambda_{n+1} x \geqq k$, we have for the series whose general term is the second term in (59)

$$
\left|\sum_{n=8+1}^{n=y} \frac{\cos n \pi c \psi\left(x, \lambda_{n}\right)}{n \sqrt{n \lambda_{n} x}}\right|<\sum_{n=8+1}^{n=\nu} \frac{K_{1}}{\sqrt{k} n^{\frac{3}{2}}}<\frac{K_{1}}{\sqrt{k}} \eta \quad\left(0<x \leqq x_{0}\right) .
$$

The first term in (59) reduces to the form

$$
\begin{aligned}
\frac{\cos n \pi c \cos n \pi x}{\sqrt{n \lambda_{n} x}} & \cos (q x-\alpha)-\frac{\cos n \pi c \sin n \pi x}{\sqrt{n \lambda_{n} x}} \sin (q x-\alpha) \\
= & \frac{\cos (q x-\alpha)}{2}\left[\frac{\cos n \pi(c+x)+\cos n \pi(c-x)}{\sqrt{n \lambda_{n} x}}\right] \\
& -\frac{\sin (q x-\alpha)}{2}\left[\frac{\sin n \pi(c+x)-\sin n \pi(c-x)}{\sqrt{n \lambda_{n} x}}\right] .
\end{aligned}
$$

* That such a positive constant exists, is easily inferred from equation (41).

$\dagger$ Cf. TANnery, Fonctions d'une variable, $\S 70$. Abel's lemma applies by virtue of the fact that $J_{\nu}\left(\lambda_{n} x\right)$ steadily decreases or steadily increases with increasing $n$ as long as $n \leqq 8$.

$\ddagger$ For the sake of convenience in writing we have replaced $(2 \nu+1) \pi / 4$ by $a$. 
But we have from (52)

$$
\begin{array}{ll}
\left|\sum_{n=s+1}^{n=\nu} \frac{\cos n \pi(c+x)}{\sqrt{n}}\right|<\eta, & \left|\sum_{n=s+1}^{n=\nu} \frac{\cos n \pi(c-x)}{\sqrt{n}}\right|<\eta, \\
\left|\sum_{n=s+1}^{n=\nu} \frac{\sin n \pi(c+x)}{\sqrt{n}}\right|<\eta, & \left|\sum_{n=s+1}^{n=\nu} \frac{\sin n \pi(c-x)}{\sqrt{n}}\right|<\eta,
\end{array}
$$

when $x$ varies in the interval $0<x \leqq x_{0}$. Hence, from the lemma of Abel referred to above,

$$
\left|\sum_{n=s+1}^{n=\nu} \frac{\cos n \pi(c+x)}{\sqrt{n \lambda_{n} x}}\right|=\frac{1}{\sqrt{\lambda_{s+1} x}}\left|\sum_{n=s+1}^{n=\nu} \frac{\cos n \pi(c+x)}{\sqrt{n}} \cdot \frac{\sqrt{\lambda_{s+1}}}{\sqrt{\lambda_{n}}}\right|<\frac{\eta}{\sqrt{k}}
$$

By the use of the other inequalities in (62) and Abel's lemma, we can obtain inequalities analogous to (63) for each of the sums

$$
\sum_{n=s+1}^{n=y} \frac{\cos n \pi(c-x)}{\sqrt{n \lambda_{n} x}}, \quad \sum_{n=s+1}^{n=\nu} \frac{\sin n \pi(c+x)}{\sqrt{n \lambda_{n} x}}, \quad \sum_{n=s+1}^{n=y} \frac{\sin n \pi(c-x)}{\sqrt{n \lambda_{n} x}} .
$$

Combining these inequalities and (63), and taking note of the fact that (61) is another way of writing the first term in (59), we obtain

$$
\left|\sum_{n=s+1}^{n=y} \frac{\cos n \pi c \cos (n \pi x+q x-\alpha)}{\sqrt{n \lambda_{n} x}}\right|<\frac{4 \eta}{\sqrt{k}} \quad\left(0<x \leqq x_{0}\right) .
$$

Since (59) is another way of writing the general term of the first series on the right hand side of (58), we have from (60), (64), and (59)

$$
\left|A \sum_{n=8+1}^{n=y} \frac{\cos n \pi c \cos \left(\lambda_{n} x-a\right)}{\sqrt{n \lambda_{n} x}}\right|<\frac{A\left(K_{1}+4\right) \eta}{\sqrt{k}}=C_{2} \eta \quad\left(0<x \leqq x_{0}\right) .
$$

We consider next the series that forms the second term on the right hand side of (58). If we set

$$
\epsilon_{n}=-\sum_{r=n}^{r=\infty} \frac{\cos r \pi c}{\sqrt{r}}
$$

we have for this series *

$$
\begin{aligned}
\sum_{n=s+1}^{n=\nu} \frac{\cos n \pi c}{\sqrt{n}} \cdot \frac{\psi\left(\lambda_{n} x\right)}{\left(\lambda_{n} x\right)^{i}} & =-\epsilon_{c+1} \frac{\psi\left(\lambda_{c+1} x\right)}{\left(\lambda_{c+1} x\right)^{i}} \\
& \quad+\sum_{n=s+1}^{n=\nu}\left(\frac{\psi\left(\lambda_{n} x\right)}{\left(\lambda_{n} x\right)^{i}}-\frac{\psi\left(\lambda_{n+1} x\right)}{\left(\lambda_{n+1} x\right)^{l}}\right) \epsilon_{n+1}+\epsilon_{\nu+1} \frac{\psi\left(\lambda_{n+1} x\right)}{\left(\lambda_{v+1} x\right)^{i}}
\end{aligned}
$$

But from the Law of the Mean

$$
\frac{\psi\left(\lambda_{n} x\right)}{\left(\lambda_{n} x\right)^{!}}-\frac{\psi\left(\lambda_{n+1} x\right)}{\left(\lambda_{n+1} x\right)^{!}}=\frac{\psi^{\prime}\left(\xi_{n} x\right)}{\xi_{n}^{\prime} x^{\frac{1}{l}}}-\frac{3 \psi\left(\xi_{n} x\right)}{2 \xi_{n}^{\prime} x^{!}} \quad\left(\lambda_{n}<\xi_{n}<\lambda_{n+1}\right) .
$$

* Cf. equation (26) of the anthor's article in these Transactions, vol. 8 (1907), p. 299. 
Moreover, in riew of (47), (18), and (55), we have

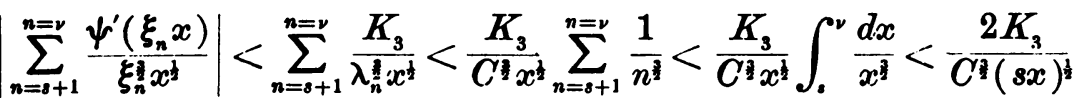

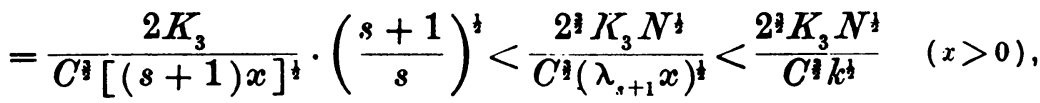

and

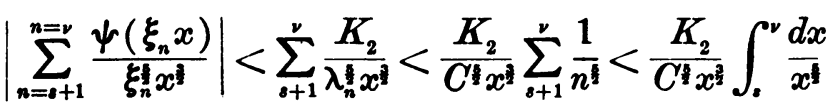

From (66) and (52) we have

$$
<\frac{2 K_{2}}{3 C^{5}(s x)^{1}}<\frac{2 ! K_{2} N^{3}}{3 C^{3} k^{3}} \quad(x>0) \text {. }
$$

Combining (67) with (68), (69), (70), (71), and (47), we obtain

$$
\left|\sum_{n=0+1}^{n=\nu} \frac{\cos n \pi c}{\sqrt{n}} \cdot \frac{\psi\left(\lambda_{n} x\right)}{\left(\lambda_{n} x\right)^{3}}\right|<\frac{2 N^{3}\left(3 C K_{3} k+2 K_{2} N\right)+6 K_{2} C}{3 C^{3} k^{3}} \eta=C_{3} \eta
$$

From (58), (65), and (72) we have

$$
\left|R_{2}\right|<\left(C_{2}+C_{3}\right) \eta
$$$$
\left(0<x \leqq x_{0}\right),
$$

which, combined with (56) and (57), gives

$$
\left|\sum_{n=\mu}^{n=\nu} \frac{\cos n \pi c J_{\nu}\left(\lambda_{n} x\right)}{\sqrt{n}}\right|<\left(1+C_{2}+C_{3}\right) \eta=\delta \quad\left(0<x \leqq x_{0}\right) .
$$

By a discussion precisely analogous to that of $R_{1}$ in Case $(A)$, we get for Case (B)

$$
\left|\sum_{n=\mu}^{n=\nu} \frac{\left.\cos n \pi c J_{\nu} \lambda_{n} x\right)}{\sqrt{n}}\right|<\eta<\delta \quad\left(0<x \leqq x_{0}\right),
$$

and by a discussion precisely analogous to that of $R_{2}$ in Case $(A)$, we get for Case $(C)$

$$
\left|\sum_{n=\mu}^{n=\nu} \frac{\cos n \pi c J_{\nu}\left(\lambda_{n} x\right)}{V \bar{n}}\right|<\left(C_{2}+C_{3}\right) \eta<\delta \quad\left(0<x \leqq x_{0}\right) .
$$

Combining (74), (75), and (76), we see that the series whose general term is the first expression in (51) converges uniformly for all values of $x$ lying in the interval $0<x \leqq x_{0}$. Since the series is also convergent for $x=0$, it follows that it is uniformly convergent in the interval $0 \leqq x \leqq x_{0}$. As we have pointed out before, the discussion of the series whose general term is the second expression in (51), is entirely analogous, and hence our lemma is to be regarded as completely established. 
LEMма 3. If the quantity (48) is written in the form

$$
\begin{aligned}
& -\frac{A}{2 \sqrt{\pi x}}\left[\begin{array}{r}
\cos \{q(x+c)-\nu \pi\} \frac{\cos n \pi(x+c)}{n}-\sin \{q(x+c)-\nu \pi\} \frac{\sin n \pi(x+c)}{n} \\
+\cos q(x-c) \frac{\sin n \pi(x-c)}{n}+\sin q(x-c) \frac{\cos n \pi(x-c)}{n}
\end{array}\right] \\
& +v_{n}(x),
\end{aligned}
$$

$v_{n}(x)$ will be the general term of a series that is uniformly convergent in any interval

$$
0<x_{0} \leqq x \leqq 1
$$

By means of $(46),(48)$ reduces to the form

$$
\begin{gathered}
\frac{\cos \left[(n \pi+q) c-\frac{2 \nu+3}{4} \pi\right] \cos \left(\lambda_{n} x-\frac{2 \nu+1}{4} \pi\right)}{\sqrt{n \lambda_{n} x}} \\
+\frac{\cos \left[(n \pi+q) c-\frac{2 \nu+3}{4} \pi\right] \psi\left(\lambda_{n} x\right)}{\sqrt{n}\left(\lambda_{n} x\right)^{\frac{3}{2}}} .
\end{gathered}
$$

The second term of (79) is obviously the general term of a series that is uniformly convergent in the interval (78); if then, by subtracting from the first term a number of quantities that have the same property, we can reduce it to an expression which is equal to the first term in (77), our lemma will be established.

From (44) we see that the first term in (79) can be written in the form

$$
\begin{gathered}
A \frac{\cos \left[(n \pi+q) c-\frac{2 \nu+3}{4} \pi\right] \cos \left[(n \pi+q) x-\frac{2 \nu+1}{4} \pi\right]}{\sqrt{n \lambda_{n} x}} \\
+A \frac{\cos \left[(n \pi+q) c-\frac{2 \nu+3}{4} \pi\right] \psi\left(x, \lambda_{n}\right)}{n v^{\prime} \overline{n \lambda_{n} x}} .
\end{gathered}
$$

Since the second term of $(80)$ is obviously the general term of a series that is uniformly convergent in the interval (78), it only remains to consider the first term. By means of (42) it can be reduced to the form

$$
\begin{array}{r}
A \frac{\cos \left[(n \pi+q) c-\frac{2 \nu+3}{4} \pi\right] \cos \left[(n \pi+q) x-\frac{2 \nu+1}{4} \pi\right]}{n \sqrt{\pi x}}+\frac{\cos \left[(n \pi+q) c-\frac{2 \nu+3}{4} \pi\right] \psi_{1}(n)}{n^{2} \sqrt{n x}} .
\end{array}
$$


Here again the second term is obviously the general term of a series that is uniformly convergent in the interval (78); if we can show further that the first term is equal to the first term of (77), our lemma will be proved.

By means of a well known trigonometric formula the first term of (81) may be reduced to the form

$$
\begin{aligned}
& A \frac{\cos [(n \pi+q)(x+c)-(\nu+1) \pi]+\cos \left[(n \pi+q)(x-c)+\frac{\pi}{2}\right]}{2 n \sqrt{\pi x}} \\
& 82) \quad=-A \frac{\cos [(n \pi+q)(x+c)-\nu \pi]+\sin (n \pi+q)(x-c)}{2 n \sqrt{\pi x}} \\
& =-\frac{A}{2 \sqrt{\pi x}}\left[\begin{array}{c}
\cos \{q(x+c)-\nu \pi\} \frac{\cos n \pi(x+c)}{n}-\sin \{q(x+c)-\nu \pi\} \frac{\sin n \pi(x+c)}{n} \\
+\cos q(x-c) \frac{\sin n \pi(x-c)}{n}+\sin q(x-c) \frac{\cos n \pi(x-c)}{n}
\end{array}\right] .
\end{aligned}
$$

The right hand side of (82) is identical with the first term of (77), and consequently our lemma is completely established.

Lemмa 4. The quantity (48) is the general term of a series for which the sum of the first $n$ terms remains finite for all values of $n$ and all values of $x$ Tying in the interval $0 \leqq x \leqq 1$.

That the sum of the first $n$ terms of this series remains finite for all values of $n$ and all values of $x$ lying in the interval $0 \leqq x \leqq x_{0}<c$, is an immediate consequence of Lemma 2. It remains to be shown that this sum remains finite for all values of $n$ and all values of $x$ lying in the interval (78).

We know from Lemma 3 that when the expression (48) is written in the form (77), $v_{n}(x)$ is the general term of a series that is uniformly convergent in the interval (78) and for which therefore the sum of the first $n$ terms remains finite for all values of $n$ and all values of $x$ in the interval (78). It only remains to be shown then that the first term of (77) is the general term of a series for which the sum of the first $n$ terms remains finite for all values of $n$ and all values of $x$ lying in the interval (78). We will prove this by showing that each term of the quantity in brackets in (77) is the general term of such a series.

The series

$$
\sum_{n=1}^{\infty} \frac{\sin n \pi z}{n}
$$

is the Fourier's development of the periodic function

$$
\begin{array}{cr}
\phi(z)=\frac{\pi-z}{2} & 0<z<2 \pi, \\
\phi(z+2 \pi)=\phi(z) & z \neq 0, \pm 2 \pi, \pm 4 \pi, \ldots
\end{array}
$$


and consequently, from a well known property of the Fourier's developments, the sum of the first $n$ terms of this series remains finite for all values of $n$ and all values of $z$. Hence the series whose general terms are the second and third terms of the expression in brackets in (77) have the property we desire to estabblish, and it only remains to examine the series whose general terms are the first and fourth terms of this expression.

If we represent by $s_{n}(z)$ the sum of the first $n$ terms of the series

we have ${ }^{*}$ for $\sin (z / 2) \neq 0$

$$
\sum_{n=1}^{\infty} \frac{\cos n z}{n}
$$

$$
s_{n}(z)=\frac{-\sin \frac{z}{2}+\sum_{m=1}^{m=n-1}\left(\frac{1}{m}-\frac{1}{m+1}\right) \sin \frac{2 m+1}{2} z+\frac{1}{n} \sin \frac{2 n+1}{2} z}{2 \sin \frac{z}{2}} .
$$

Consequently

$$
\left|s_{n}(z)\right|<\frac{1+\sum_{m=1}^{m=n-1}\left(\frac{1}{m}-\frac{1}{m+1}\right)+\frac{1}{n}}{2\left|\sin \frac{z}{2}\right|}=\frac{1}{\left|\sin \frac{z}{2}\right|}
$$

and hence we have for the sum of the first $n$ terms of the series whose general term is the fourth term of the expression in brackets in (77)

$$
\left|s_{n}(x)\right|<\frac{|\sin q(x-c)|}{\left|\sin \frac{1}{2} \pi(x-c)\right|} .
$$

It follows from the last inequality that the sum of the first $n$ terms of this series remains finite for all values of $n$ and all values of $x$ lying in the interval (78).

We have finally to consider the series whose general term is the first term of the expression in brackets in (77). In view of (85) we have for the first $n$ terms of this series

$$
\left|s_{n}(x)\right|<\frac{|\cos \{q(x+c)-\nu \pi\}|}{\left|\sin \frac{1}{2} \pi(x+c)\right|}
$$

In order to assure ourselves that $s_{n}(x)$ remains finite for all values of $n$ and all values of $x$ lying in the interval (78) we must examine the values that $q$ may take on. We have $\dagger$

$$
q=\frac{\pi}{2}+\frac{2 \nu+1}{4} \pi+k_{1} \pi \quad \text { or } \quad q=\frac{2 \nu+1}{4} \pi+k_{2} \pi
$$

* Cf. the writer's paper, these Transactions, vol. 10 (1909), p. 408, equation (66).

$\dagger$ Loc. oit., p. 415. The $\alpha$ in these values of $q$ was used, for the sake of simplicity, in place of the quantity $(2 \nu+1) \pi / 4$. 
where $k_{1}$ and $k_{2}$ are positive or negative integers or zero. Consequently, even for $c=1$, the expression on the right hand side of (86) remains finite for all values of $x$ lying in the interval (78), and hence the same is true of $s_{n}(x)$.

We have now shown that each term of the expression in brackets in (77) is the general term of a series for which the sum of the first $n$ terms remains finite for all values of $n$ and all values of $x$ in the interval (78). Hence the whole first term of (77) is the general term of such a series and our lemma is proved.

Lemma 5. The expression (48) is, for the case $c<1$, the general term of a series that is uniformly convergent in any interval

$$
c<x_{1} \leqq x \leqq 1 ;
$$

for the case $c=1$, it is the general term of a series that is uniformly convergent in any interval

$$
0<x_{1} \leqq x \leqq 1,
$$

provided the $l$ of equation (2) is not zero.

Let us consider first the case where $c<1$. We know from Lemma 3 that when (48) is written in the form (77), $v_{n}(x)$ is the general term of a series that is uniformly convergent in the interval (88). It remains to be shown that the first term of (77) is also the general term of a series that is uniformly convergent in this interval.

The series

$$
\sum_{n=1}^{\infty} \frac{\sin n z}{n}, \quad \sum_{n=1}^{\infty} \frac{\cos n z}{n}
$$

converge uniformly * throughout the interval

$$
\delta_{1} \leqq z \leqq 2 \pi-\delta_{2},
$$

where $\delta_{1}$ and $\delta_{2}$ are fixed positive quantities, which may be taken as small as we please. Hence each term of the quantity in brackets in (77) is the general term of a series that is uniformly convergent in the interval (88). Therefore the whole first ,term is the general term of such a series, and our lemma is proved for the case where $c<1$.

We turn now to the case $c=1, l \neq 0$. As in the previous case, we may, by the use of Lemma 3, reduce the proof of our lemma to the proof of the fact that the first term of (77) is the general term of a series that is uniformly convergent in the interval in question. We will now establish this fact by showing that for the values that $c$ and $l$ have in the case we are considering, this term is identically zero. Thus our lemma will be completely proved.

* Cf. BûCHer, loc. oit., p. 111. 
Since the $l$ of equation (2) is not zero, we have for $q$ [cf. the footnote to (87)]

$$
q=k_{2} \pi+\frac{2 \nu+1}{4} \pi \text {. }
$$

Moreover, we saw in the course of the proof of Lemma 3 that the first term in (81) was identical with the first term in (77). If we substitute in the first term of (81) the value $c=1$ and the value of $q$ given by (90), we find that the first factor in the numerator of this term vanishes identically. Hence the first term of (77) vanishes for the values of $l$ and $c$ in question and, as pointed out before, our lemma is proved.

Lемма 6. The expression

$$
\frac{1}{\lambda_{n}} J_{\nu}\left(\lambda_{n} x\right)
$$

where $\nu>0$, is the general term of a series for which the sum of the first $n$ terms remains finite for all values of $n$ and all values of $x$ lying in the interval

$$
0 \leqq x \leqq 1
$$

The theorem is obvious for $x=0$, since all the terms of the series vanish in that case. Hence it only remains to establish it for the interval

$$
0<x \leqq 1 \text {. }
$$

Let $\alpha_{1}$ and $\alpha_{2}$ be the smallest positive roots of

$$
\frac{d}{d x}\left\{\frac{J_{\nu}(x)}{x}\right\}=0
$$

and $J_{\nu}(x)$ respectively, and let $k$ be the smaller of the two quantities $\alpha_{1}, \alpha_{2}$. Now choose a positive integer $q$ such that $\lambda_{q}>k$ and

$$
\frac{\lambda_{m+1}}{\lambda_{m-1}}<2 \quad(m \geqq q) .
$$

From (15) we have

$$
\left|J_{\nu}\left(\lambda_{n} x\right)\right|<\begin{gathered}
N \\
\lambda_{n}^{\frac{1}{2}} x^{\frac{1}{2}}
\end{gathered}
$$

where $N$ is a positive constant. Hence for values of $x$ lying in the interval

$$
\underset{\lambda_{q}}{k} \leqq x \leqq 1,
$$

the series whose general term is (91) is uniformly convergent, and consequently the sum of the first $n$ terms of this series remains finite for all values of $n$ and all values of $x$ lying in this interval. If we can show further that the sum of 
the first $n$ terms of the series remains finite for all values of $n$ and all values of $x$ lying in the interval

$$
0<x<\frac{k}{\lambda_{q}}
$$

our lemma will be completely established.

Let us consider, then, any value of $x$ lying in the interval (98), and let $p$ be the largest positive integer such that $\lambda_{p} x<k$. There will be two cases

$$
\text { (A) } p \leqq n, \quad(B) p>n \text {. }
$$

Consider first Case $(A)$ and let us write

We have from (96)

$$
\sum_{m=1}^{m=n} \frac{J_{\nu}\left(\lambda_{m} x\right)}{\lambda_{m}}=\sum_{m=1}^{m=p-1}+\sum_{m=p}^{m=n}=S_{1}+S_{2}
$$

$$
\left|S_{2}\right|<\sum_{m=p}^{m=n} \frac{N}{\lambda_{n}^{\frac{3}{2} x^{\frac{1}{2}}}}<\frac{N}{\gamma x^{\frac{1}{2}}} \sum_{m=p}^{m=n} \frac{1}{\lambda_{m}^{\frac{1}{2}}}\left(\lambda_{m}-\lambda_{m-1}\right),
$$

where $\gamma$ is the lower limit of the distances between two successive positive roots of equation (2).* But since $\lambda_{q} x<k$ for values of $x$ in the interval (98), and since $p$ is the greatest integer such that $\lambda_{p} x<k$, it follows that $p \geqq q$, and we have from (95)

$$
\sum_{m=p}^{m=n} \frac{1}{\lambda_{m}^{\frac{3}{3}}}\left(\lambda_{m}-\lambda_{m-1}\right)<\int_{\lambda_{p-1}}^{\lambda_{n}} \frac{d x}{x^{\frac{3}{2}}}<\frac{2}{\sqrt{\lambda_{p-1}}}=\frac{2}{\sqrt{\lambda_{p+1}}} \sqrt{\frac{\lambda_{p+1}}{\lambda_{p-1}}}<\frac{2}{\sqrt{\lambda_{p+1}}} .
$$

Combining (100) and (101), we obtain, since $\lambda_{p+1} x \geqq k$,

$$
\left|S_{2}\right|<\frac{N \cdot 2^{3}}{\gamma \sqrt{\lambda_{p+1} x}} \leqq \frac{2^{3} N}{\gamma \sqrt{k}} \quad\left(0<x<\frac{k}{\lambda_{q}}\right) .
$$

The discussion of $S_{1}$ is slightly different in the two cases $\nu>1$ and $0<\nu \leqq 1$. Consider first the case $\nu>1$. We have

$$
S_{1}=\sum_{m=1}^{m=p-1} \frac{J_{\nu}\left(\lambda_{m} x\right)}{\lambda_{m} x} x \leqq \frac{1}{\gamma} \sum_{m=1}^{m=p-1} \frac{J_{\nu}\left(\lambda_{m} x\right)}{\lambda_{m} x}\left(\lambda_{m+1}-\lambda_{m}\right) x
$$

But since, for $\nu>1$, the function $J_{\nu}(y) / y$ is positive and steadily increases in the interval $0<y<k$, where $k$ has the same meaning as above, we have

$$
\sum_{m=1}^{m=p-1} \frac{J_{\nu}\left(\lambda_{m} x\right)}{\lambda_{m} x}\left(\lambda_{m+1} x-\lambda_{m} x\right)<\int_{0}^{\lambda_{p} x} \frac{J_{\nu}(y)}{y} d y<\int_{0}^{k} \frac{J_{\nu}(y)}{y} d y .
$$

Combining (103) and (104), we obtain

$$
0<S_{1}^{\curlyvee}<\frac{1}{\gamma} \int_{0}^{k} \frac{J_{\nu}(y)}{y} d y \quad\left(\nu>1 ; 0<x<\frac{k}{\lambda_{q}}\right) .
$$

* That such a lower limit exists and is different from zero is easily inferred from the fact that the limit of the distance between the $n$th and the $(n+1)$ th roots of $(2)$ exists and is equal to $\pi$.

Trans. Am. Math. Soc. 14 
Taking up next the case $0<\nu \leqq 1$, we have

$$
S_{1}=\sum_{m=1}^{m=p-1} \frac{J_{\nu}\left(\lambda_{m} x\right)}{\lambda_{m} x} x<\frac{1}{\gamma_{1}} \sum_{m=1}^{m=p-1} \frac{J_{\nu}\left(\lambda_{m} x\right)}{\lambda_{m} x}\left(\lambda_{m}-\lambda_{m-1}\right) x
$$

where we have set $\lambda_{0}=0$ for the sake of uniformity, and have used $\gamma_{1}$ to represent the smaller of the two quantities $\gamma$ and $\lambda_{1}$. But since, for $0<\nu \leqq 1$, the function $J_{v}(y) / y$ is positive and steadily decreases in the interval $0<y<k$, where $k$ has the same meaning as above, we have

$$
\sum_{m=1}^{m=p-1} \frac{J_{\nu}\left(\lambda_{m} x\right)}{\lambda_{m} x}\left(\lambda_{m} x-\lambda_{m-1} x\right)<\int_{0}^{\lambda_{p-1} x} \frac{J_{\nu}(y)}{y} d y<\int_{0}^{k} \frac{J_{\nu}(y)}{y} d y,
$$

and hence, combining (107) with (106),

$$
0<S_{1}<\frac{1}{\gamma_{1}} \int_{0}^{k} \frac{J_{\nu}(y)}{y} d y \quad\left(0<\nu \leqq 1 ; 0<x<\frac{k}{\lambda_{q}}\right) .
$$

From (105) and (108) we have

$$
0<S_{1}<K
$$$$
\left(v>0 ; 0<x<\frac{k}{\lambda_{q}}\right) \text {, }
$$

where $K$ is a positive constant. Combining (99), (102), and (109), we obtain

$$
\left|\sum_{m=1}^{m=n} \frac{J_{\nu}\left(\lambda_{m} x\right)}{\lambda_{m}}\right|<K+\frac{2 i N}{\gamma \sqrt{k}} \quad\left(0<x<\frac{k}{\lambda_{q}}\right),
$$

and hence in Case $(A)$ the sum of the first $n$ terms of the series whose general term is (91) remains finite for all values of $n$ and all values of $x$ in the interval (98). The treatment of Case $(B)$ is entirely analogous to the treatment of $S_{1}$ in Case $(A)$, and consequently we may infer that the sum of the first $n$ terms of the series remains finite for all values of $n$ and all values of $x$ in the interval (98). Hence, as was pointed out before, our lemma is proved.

Theorem I. If $f(x)$ satisfies the conditions of Lemma 1, the series (6) will, provided $\nu=0$ or $f(0)=0$, converge uniformly to $f(x)$ throughout any interval

$$
0 \leqq x \leqq x_{0}<c_{1},
$$

where $c_{1}$ is the smallest value of $x$ for which $f(x)$ has a discontinuity; provided the $l$ of equation (7) is not zero or $f(1)$ is zero, it will converge uniformly to $f(x)$ throughout any interval

$$
c_{k}<x_{1} \leqq x \leqq 1,
$$

where $c_{k}$ is the largest value of $x$ for which $f(x)$ has a discontinuity ; finally, the sum of $n$ terms of the series will remain finite for all values of $n$ and all values of $x$ lying in the interval

$$
0 \leqq x \leqq 1
$$


In view of Lemma 1 , the general term of the series $(1)$, and hence the general term of the series (6),* may be written in the form

$$
\begin{gathered}
\sum_{m=1}^{m=k} M_{m} \frac{\cos \left[(n \pi+q) c_{m}-\frac{2 \nu+3}{4} \pi\right]}{\sqrt{n}} J_{\nu}\left(\lambda_{n} x\right) \\
+M f(1) \frac{\cos \left[(n \pi+q)-\frac{2 \nu+3}{4} \pi\right]}{\sqrt{n}} J_{\nu}\left(\lambda_{n} x\right) \\
+\frac{\nu K f(0)}{\lambda_{n}} J_{\nu}\left(\lambda_{n} x\right)+r_{n} J_{\nu}\left(\lambda_{n} x\right),
\end{gathered}
$$

where the $M$ 's and $K$ are constants, the $c$ 's are points of discontinuity of $f(x)$, and $r_{n}$ is the general term of an absolutely convergent series.

If either $\nu=0$ or $f(0)=0$, the third term in (114) drops out, and it only remains to consider the other three terms. Since the Bessel function of any order remains finite for all values of the argument, it is obvious that the last term in (114) is the general term of a series that is uniformly convergent in the interval (111). It follows from Lemma 2 that the first and second terms of (114) are also the general terms of series that are uniformly convergent in this interval. Hence the whole expression (114) is the general term of such a series, and since (114) is only another way of writing the general term of (6), it follows that (6) is uniformly convergent in the interval (111). Since this is the case and since the terms of (6) are continuous in this interval, it must define there a continuous function of $x, \chi(x)$. However, for values of $x$ lying in the interval $0<x \leqq x_{0}$, (6) converges to $f(x),+$ i. e.,

$$
\chi(x)=f(x) \quad\left(0<x \leqq x_{0}\right) .
$$

Both sides of the last equation have a limit as $x$ approaches zero. Equating these limits, we get

$$
\chi(0)=f(0) \text {. }
$$

Hence (1) converges uniformly to $f(x)$ throughout the interval (111), provided $\nu=0$ or $f(0)=0$, and the first part of our theorem is proved.

We wish next to show that, provided the $l$ of equation (7) is not zero, or $f(1)$ is zero, the series (6) will converge uniformly to $f(x)$ throughout the interval (112).

Consider first the case $l \neq 0$. We saw in the previous discussion that the general term of (6) could be written in the form (114). It is obvious that the

\footnotetext{
* In the special case where (4) holds, $(114)$ is the $(n+1)$ th term of $(6)$, but that of course makes no difference in a discussion of convergence.

† This, as we have stated earlier in this paper, has been established in previous discussions of the series (6). Cf. the artioles by DINI, KNBSER, and Hobson quoted above.
} 
third and fourth terms of (114) are the general terms of series that are uniformly convergent in the interval (112); it follows from Lemma 5 that the first term of (114) and, since the $l$ of equation (7) is not zero, the second term of (114) are the general terms of series that are uniformly convergent in this interval. Hence the whole expression, $(114)$ is the general term of such a series, and since (114) is only another way of writing the general term of (6), it follows that (6) is uniformly convergent in the interval (112), provided the $l$ of equation ( 7 ) is not zero.

Considering next the case of $f(1)=0$, we see that the second term of (114) is zero for this case, and is therefore the general term of a series that is uniformly convergent in the interval (112), whether the $l$ of equation (7) is or is not zero. Hence, in this case also, (6) is uniformly convergent in the interval (112).

That the series (6) has the value $f(x)$ in this interval, under the conditions. prescribed, was proved by HoBson in the paper already quoted.

We come finally to the proof of the third part of our theorem, namely that the sum of $n$ terms of the series (6) remains finite for all values of $n$ and all values of $x$ lying in the interval (113). Since $r_{n}$ is the general term of an absolutely convergent series, the fourth term of (114) is obviously the general term of a series that is absolutely and uniformly convergent in the interval (113), and for which therefore the sum of $n$ terms remains finite for all values of $n$ and all values of $x$ lying in this interval. It follows at once from Lemma 4 that the first and second terms of (114) are also the general terms of such series, and from Lemma 6 that the third term is the general term of such a series. Hence the whole expression (114), or its equivalent the general term of (6), is the general term of such a series.

Our theorem is, therefore, completely established.

The preceding theorem establishes the uniform convergence of the series (6) to $f(x)$ throughout the interval (111) only when $\nu=0$ or $f(0)=0$. That (6) could not possibly converge uniformly to $f(x)$ in the interval (111) in case neither of these restrictions was fulfilled is easy to see, since when $\nu>0$ the value of (6) for $x=0$ is always zero. However, in the case $\nu>0, f(0) \neq 0$, it is possible to obtain a development of $f(x)$ that is uniformly convergent in the interval (111), in the form of a constant term plus a series of the type (6). To do this, we have only to develop the function $\phi(x)=f(x)-f(0)$ in a series of the form (6), and precede this development by the constant term $f(0)$.

The reasoning in Theorem $I$ is also immediately applicable to proving that under the given conditions on $f(x)$ the series (6) will converge uniformly in any closed sub-interval of $0<x<1$ that does not include a point of discontinuity of $f(x)$. This uniform convergence has been previously established,*

* By KNRSER for developments in terms of $J_{0}$; by HoBson for developments in terms of $J_{\nu}(\nu \geqq 0)$. Cf. the references given above. 
but it seems worth while to point out the fact that it can be obtained independently from the results of the present paper.

TheOREM II. If $f(x)$ satisfies the conditions of Lemma 1 , the series

$$
A_{1} e^{-\lambda_{1} z} F_{0}\left(\lambda_{1}, x\right)+A_{2} e^{-\lambda_{2} z} F_{0}\left(\lambda_{2}, x\right)+\cdots,
$$

where the A's are the coefficients of the series (6) for the case $\nu=0$, and the $\lambda$ 's are the roots, positive or zero, of equation (7) arranged in increasing order of magnitude, will converge absolutely in the region

$$
\left(\begin{array}{c}
0 \leqq x \leqq 1 \\
z>0
\end{array}\right)
$$

its value $u(z, x)$ will be continuous there, will approach $f(x)$ as we approach any point of the interval $0 \leqq x<1$ lying on the line $z=0$, at which $f(x)$ is continuous, will approach $f(1)$ as we approach the point $x=1, z=0$, if the $l$ of equation (7) is not zero and $f(x)$ is continuous at $x=1$, and finally will remain finite when we approach any point of the interval $0 \leqq x \leqq 1$ lying on the line $z=0$.

We obtain at once from the definition of $A_{n}$ and Lemma 1

$$
\left|A_{n}\right|=\left|A_{n}^{\prime}\right|<K_{1}
$$$$
(n=1,2, \cdots),
$$

where $K_{1}$ is a positive constant. Then since $F_{0}(\lambda, x)$ remains finite for all values of $x$ and $\lambda$, we have for the general term of (115), if we make use of (18), $\left|A_{n} e^{-\lambda_{n} z} F_{0}\left(\lambda_{n}, x\right)\right|<\frac{K_{1}\left|F_{0}\left(\lambda_{n}, x\right)\right|}{e^{\lambda_{n} z}}<\frac{K}{e^{\lambda_{n} z_{0}}}<\frac{K}{\left(\lambda_{n} z_{0}\right)^{2}}<\frac{K}{C^{2} z_{0}^{2} n^{3}} \quad\left(z \geqq z_{0}>0\right)$, where $K$ is a positive constant. Hence (115) converges absolutely and uniformly for all values of $x$ and $z$ lying in the region

$$
\left(\begin{array}{l}
0 \leqq x \leqq 1 \\
z \geqq z_{0}>0
\end{array}\right) .
$$

Since also its terms are continuous in this region, it represents a function that is continuous there or, since $z_{0}$ may be taken as any constant $>0$, is continuous in the region (116).

At any point of the interval $0 \leqq x<1$ at which $f(x)$ is continuous, and at the point $x=1$ when the $l$ of equation (7) is not zero and $f(x)$ is continuous there, the series (6) converges to $f(x)$. Hence, for values of $x$ in the interval $0 \leqq x<1$ at which $f(x)$ is continuous, and for $x=1$ in the case $l \neq 0$ and $f(x)$ is continuous at $x=1, u(z, x)$ will approach $f(x)$ as $z$ approaches +0 , since the convergence factors

$$
e^{-\lambda_{1} z}, e^{-\lambda_{2} z}, \cdots
$$


satisfy the conditions of a theorem due to Bromwich.* Furthermore, (6) converges uniformly to $f(x)$ throughout any closed interval, in which $f(x)$ is continuous, that lies in the interval $0 \leqq x \leqq 1$ and does not include the point $x=1$ for the case where the $l$ of equation (7) is zero. Consequently $u(z, x)$ will approach $f(x)$ uniformly for all values of $x$ lying in such an interval as $z$ approaches +0 , as can be shown by a slight modification of the reasoning in Bromwich's theorem. Hence $u(z, x)$ will approach $f(x)$ as we approach any point of the interval $0 \leqq x \leqq 1$ lying on the line $z=0$ at which $f(x)$ is continuous, $\dagger$ along any path lying in the region (116).

It remains finally to be shown that $u(z, x)$ remains finite as we approach any point of the interval $0 \leqq x \leqq 1$ lying on the line $z=0$.

If we represent by $s_{n}(x)$ the sum of the first $n$ terms of the series (6), we may write the series (115) in the form

$$
\sum_{n=1}^{\infty}\left[s_{n}(x)-s_{n-1}(x)\right] e^{-\lambda_{n} z},
$$

where for the sake of uniformity we have taken $8_{0}(x)=0$. We know from Theorem I that $s_{n}(x)$ remains finite for all values of $n$ and all values of $x$ lying in the interval (113). Consequently, since for $z>0$

$$
\lim _{n=\infty} e^{-\lambda_{n} z}=0
$$

the series obtained by dropping the parentheses in the series (118) is one for which the limit of the general term is zero. Hence, since no parenthesis contains more than a fixed number of terms, we may drop the parentheses without changing the value of the series. Rearranging the terms in such a manner that

* Mathematische Annalen, vol. 65 (1908), p. 358. BromwrCh's conditions are

$$
\left.\begin{array}{l}
\sum_{n=\mu}^{n=v} n k\left|\Delta^{k+1} v_{n}\right|<K \\
\lim _{n=\infty} n^{k} v_{n}=0 \\
\lim _{a=+0} v_{n}=1,
\end{array}\right\} a>0,
$$

where $\mu$ and $\nu$ are two integers, $v_{1}, v_{2}, v_{3}, \cdots$ are the convergence factors, $\Delta^{k+1} v_{n}$ represents their $(k+1)$ th differences, and $K$ is a positive constant. When the convergence factors are introduced into a convergent series, as in the present instance, the $k$ of this theorem must be taken equal to zero.

A simple prool of the special case here dealt with of BromwrCh's theorem can be obtained from the expression (119), which is equal to the series (115), by throwing it into the form

where

$$
f(x) e^{-\lambda_{1} z}+\sum_{n=1}^{n=\infty} \varepsilon_{n}(x)\left[e^{-\lambda_{n} z}-e^{-\lambda_{n+1} z}\right],
$$

$$
\varepsilon_{n}(x)=8_{n}(x)-f(x) \text {. }
$$

† We must except, of course, the point $x=1$ for the case where the $l$ of equation (7) is equal to zero. 
no term is carried over more than a fixed number of terms and reinserting parentheses does not change the value of the series, and hence (118) may be written in the form

But since

$$
\sum_{n=1}^{\infty} s_{n}(x)\left[e^{-\lambda_{n} z}-e^{-\lambda_{n+1} z}\right]
$$

$$
\left|s_{n}(x)\right|<M \quad(n=1,2, \cdots ; 0 \leqq x \leqq 1),
$$

where $M$ is a positive constant, we have, in view of the fact that (119) converges to $u(z, x)$,

$$
|u(z, x)| \leqq \sum_{n=1}^{\infty}\left|s_{n}(x)\right| \cdot\left[e^{-\lambda_{n} z}-e^{-\lambda_{n+1} z}\right]<M \sum_{n=1}^{\infty}\left[e^{-\lambda_{n} x}-e^{-\lambda_{n+1} z}\right]=M e^{-\lambda_{1} z},
$$

and consequently $u(z, x)$ remains finite when we approach any point of the interval $0 \leqq x \leqq 1$ lying on the line $z=0$.

Our theorem is, therefore, completely established.

A single example will show clearly the manner in which the above results can be applied to problems in mathematical physics. Let us consider the problem of determining the steady flow of heat in a semi-infinite circular cylinder whose base is kept at a temperature

$$
v=f(r)
$$

where $r$ is the distance from the axis of the cylinder.

This problem reduces to the determination of a function $v(r, z)$ which is finite throughout the region $z \geqq 0,0 \leqq r \leqq 1$, and is continuous throughout this region except when $z=0$ and $r$ has a value for which $f(r)$ is discontinuous; which satisfies the equation

in the region

$$
\frac{1}{r} \frac{\partial}{\partial r}\left(r \frac{\partial v}{\partial r}\right)+\frac{\partial^{2} v}{\partial z^{2}}=0
$$

$$
\left(\begin{array}{c}
z>0 \\
0<r<1
\end{array}\right)
$$

and which also satisfies the conditions

$$
l \frac{\partial v}{\partial r}+h v=0 \quad(z>0, r=1)
$$

$$
v=f(r)
$$$$
(z=0) .^{*}
$$

It can be shown easily that the function

$$
A e^{-\lambda \varepsilon} F_{0}(\lambda, r),
$$

where $A$ is a constant and $\lambda$ is a root of equation (7), satisfies (120) and (122).

\footnotetext{
* Cr. Carglaw, Introduction to the Theory of Fourier's Series and Integrals, etc., Chap. XV, p. 321.
} 
It remains to construct from functions of the form (124) a continuous function which will also satisfy (120) and (122) and in addition will approach the boundary value $f(r)$, where $f(r)$ is continuous, and which will remain finite where that is not the case.

Consider the series

$$
A_{1} e^{-\lambda_{1} z} F_{0}\left(\lambda_{1}, r\right)+A_{2} e^{-\lambda_{2} z} F_{0}\left(\lambda_{2}, r\right)+\cdots,
$$

where the $\lambda$ 's are the roots, positive or zero, of equation (7), arranged in increasing order of magnitude, and the $A$ 's are the coefficients of the series (6) for $\nu=0$ corresponding to the given function $f(r)$. It follows from Theorem II that if $f(r)$ satisfies the conditions imposed on $f(x)$ in Lemma 1 , this series will converge in the region

$$
\left(\begin{array}{c}
z>0 \\
0 \leqq r \leqq 1
\end{array}\right)
$$

and define there a continuous function $u(r, z)$; that this function satisfies (120) and (122) can be shown in a manner similar to that in which its convergence and continuity were established. Finally it follows from Theorem II that $u(r, z)$ will approach $f(r)$ when we approach a point on the line $z=0$ at which $f(r)$ is continuous,* and that it will remain finite when we approach any other point of this line. Thus $u(r, z)$ forms a complete solution of the physical problem. $\dagger$

The Univergity of Cincinnati, Cinctinatit, OHio.

* We must except here the point $r=1$ for the case in which the $l$ of equation (7) is zero. The necessity for this exception is now apparent from the physical point of view as well as the mathematical one, for the case $l=0$ corresponds to the case in which the convex surface of the oylinder is kept at temperature zero, and hence we could not approach the temperature at the ciroumference of the base of the oylinder as we approached this circumference along any path except in the very special case that this temperature was zero.

In the discussion of other problems of mathematical physics that involve developments in Bessel functions of order zero, we may encounter convergence factors slightly different from those of Theorem II. However, in most cases the necessary modifications in Theorem II will be only those of form, since the convergence faotors will satisfy the same general theorem that was used in the prool of Theorem II. 